\title{
Using Visualization in Cockpit Decision Support Systems
}

\author{
Cecilia R. Aragon \\ Lawrence Berkeley National Laboratory \\ University of California \\ Berkeley, CA 94720 \\ aragon@sims.berkeley.edu
}

\begin{abstract}
In order to safely operate their aircraft, pilots must make rapid decisions based on integrating and processing large amounts of heterogeneous information. Visual displays are often the most efficient method of presenting safety-critical data to pilots in real time. However, care must be taken to ensure the pilot is provided with the appropriate amount of information to make effective decisions and not become cognitively overloaded. The results of two usability studies of a prototype airflow hazard visualization cockpit decision support system are summarized. The studies demonstrate that such a system significantly improves the performance of helicopter pilots landing under turbulent conditions. Based on these results, design principles and implications for cockpit decision support systems using visualization are presented.
\end{abstract}

Keywords: Visualization, decision support, airflow, aviation, rotorcraft, safety.

\section{Introduction}

The dangers that invisible airflow hazards pose to aircraft may be mitigated by new hardware developments that can provide airflow data into the cockpit. The challenge then becomes how to use this data in support of pilot decisions. We discuss the process of user-centered design by which a prototype of a visual airflow hazard detection system was developed. We then present the results of two usability studies of our system, which visually displays to pilots the most critical information about the location and severity of nearby airflow hazards.

\subsection{Background}

Many aircraft accidents are caused by encounters with unseen airflow hazards near the ground, such as vortices, downdrafts, low level wind shear, microbursts, or turbulence from surrounding vegetation or structures near the landing site. These hazards can be dangerous even to airliners; there have been hundreds of fatalities in the United States in the last two decades attributable to airliner encounters with microbursts and low level wind shear alone [1,2]. Accidents often result when pilots make faulty decisions based upon insufficient knowledge of the airflow near their landing site [3].
Airflow hazards are difficult to identify simply because air is invisible. Pilots cannot visually detect airflow patterns unless the air happens to pick up dust, smoke or other aerosols that are visible to the human eye. Being thus unable to discern a factor of potentially great importance to them, pilots learn to use their intuition concerning airflow over obstacles near their takeoff or landing sites, and they learn to pick up visual cues from the surrounding area. However, airflow-related accidents still occur.

Because airflow hazards are localized in space and therefore can be avoided by pilot maneuvering, a system that could convey the hazard location and severity to the pilot could have a significant benefit to aviation safety.

\subsection{Shipboard rotorcraft operations}

Helicopters are especially vulnerable to airflow disturbances; first, by the nature of the aerodynamic forces involved, and second, because helicopters are often called upon to operate into and out of areas where obstacles surround the landing site. Whenever air flows past vegetation or sharp-edged structures, vortices and turbulence form (Figure 1).

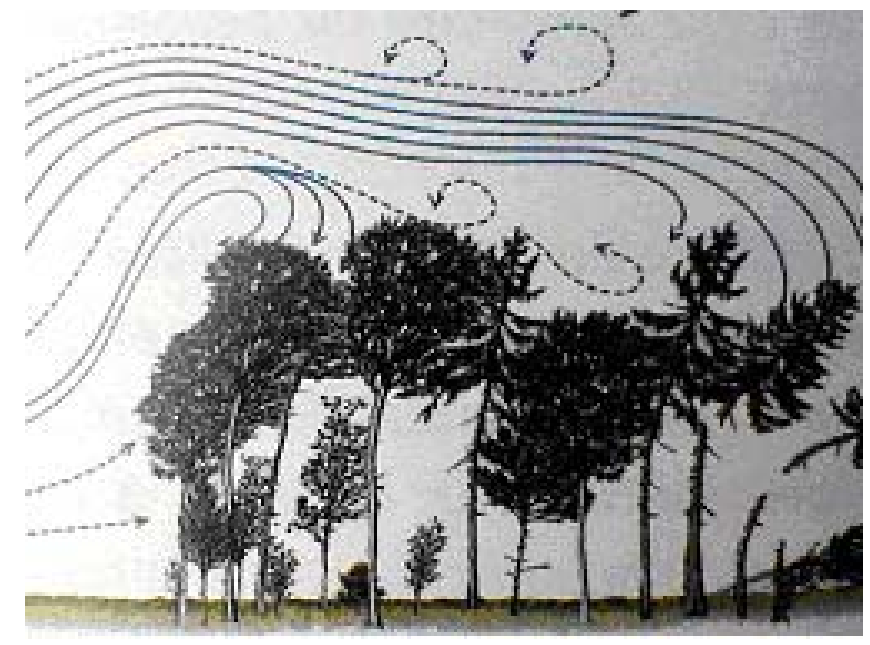

Figure 1. Turbulent airflow over vegetation (US Govt. image, http://www.nws.noaa.gov)

For example, emergency search and rescue may have to operate in mountainous areas and small clearings surrounded by vegetation where wind speeds are usually 
high. Helicopters also must land on urban rooftops, offshore oil platforms, or on the decks of ships. A device for detecting airflow hazards therefore has special utility for helicopter operations.

Landing a helicopter on a moving aircraft carrier is one of the most challenging tasks a helicopter pilot can face [4]. Because of the movement of the ship, its superstructure will always generate disturbed airflow such as vortices and turbulence. In addition, high seas may cause extreme ship pitch and roll (Figure 2), and low visibility may degrade visual cues. The pilot must maneuver the helicopter within very tight tolerances to avoid striking ship structures or other aircraft; in some cases, the rotor blades may be only a few feet from the shipboard hangar doors. It is a task that demands maximal concentration and skill from the pilot. A system that can deliver even a small amount of assistance to the pilot in this environment could have a substantial safety impact.

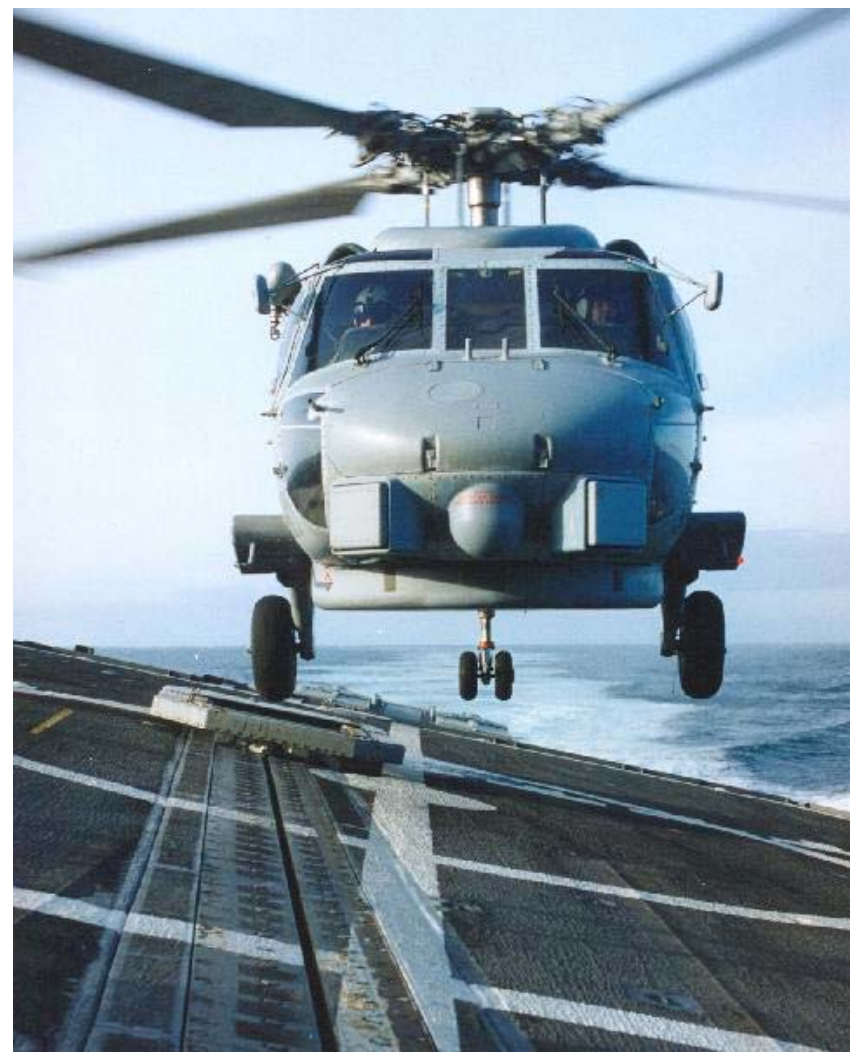

Figure 2. Helicopters landing on shipboard have to contend with high levels of pitch and roll (US Govt. image, courtesy of K. Long)

Helicopter accidents and incidents that occur on shipboard range from incidents such as "tunnel strikes" (where wind conditions can cause a helicopter's rotor blades to spin out of control, damaging the fuselage of the helicopter) to fatal accidents. Over 120 tunnel strikes have occurred in dual-rotor helicopters since the 1960s, causing damage ranging from $\$ 50 \mathrm{~K}$ to over $\$ 1 \mathrm{M}$ per incident (Figure 3)[5]. Analysis of these accidents and incidents frequently finds airflow hazards to be the root cause. The pilots and ground crew are usually unaware of the danger, and the pilot is unable to react in time [3]. Enabling the flight crew to make correct decisions in advance of the hazard encounter, therefore, could reduce or prevent such accidents.

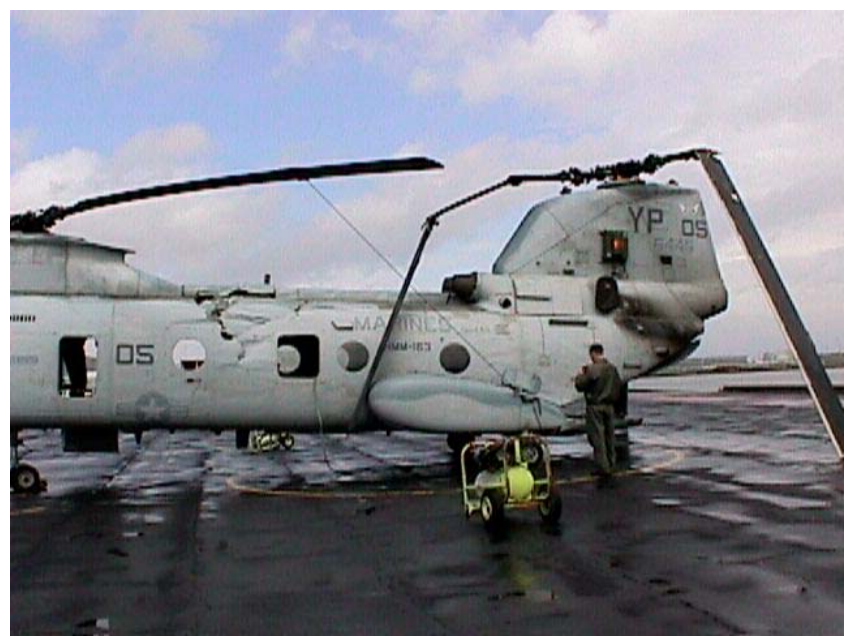

Figure 3. H-46 tunnel strike (US Govt. image, courtesy of K. Long)

The US Navy has compiled extensive amounts of data from shipboard flight tests, wind tunnel studies, computational fluid dynamics calculations, and sampling of the airflow vector velocities at various points in the flow field behind the superstructure in the helicopter landing zones with handheld anemometers. Navy flight test engineers study the airflow around moving ships of all types, and how the airwake changes when helicopters of different makes and models land on the ships. The test engineers understand the nature of ship airwake extremely well; the important concern is how best to communicate this information to fleet pilots.

Currently, the Navy produces operational envelopes (Figure 4) for each ship-rotorcraft combination [6]. For each model of helicopter and type of ship, these envelopes depict the allowable wind speeds and directions where it is considered safe to operate the helicopter. If the winds over deck are not within the envelope, the pilot is not even allowed to commence a landing approach. 


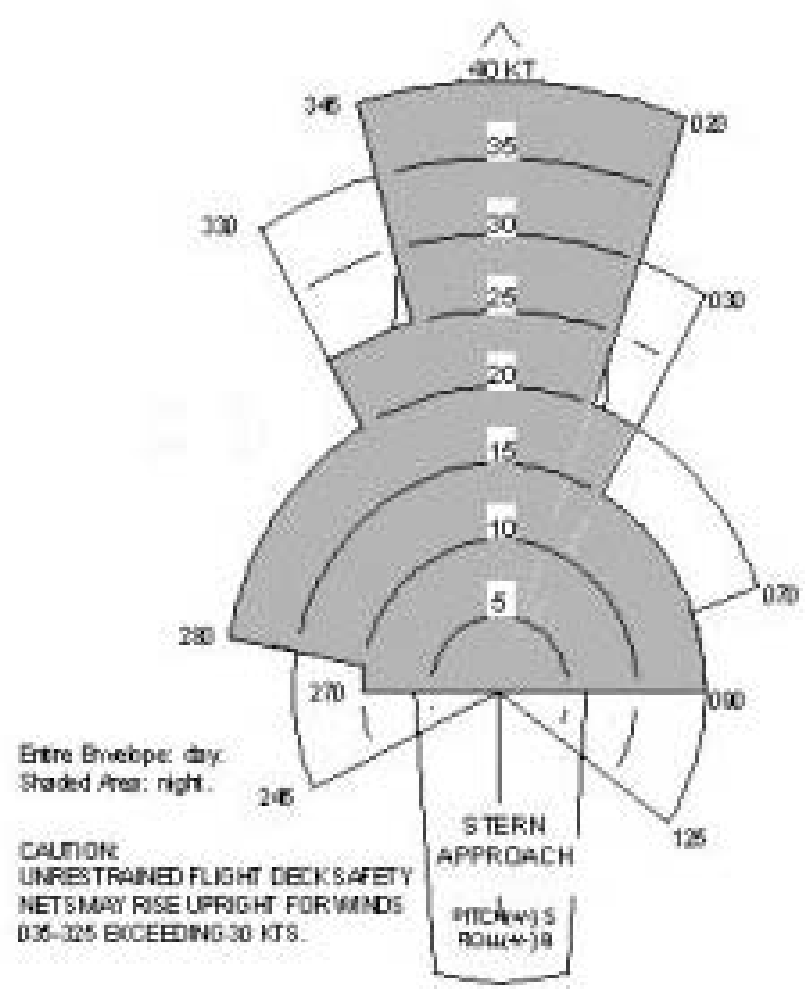

Figure 4. Shipboard rotorcraft operational envelope (US Govt. image, courtesy of K. Long)

These envelopes are necessarily conservative, as they have to include all flight conditions and all fleet pilot skill levels. The envelopes limit permitted operational conditions significantly; however, even with this cautious approach, accidents due to airflow hazards still occur.

\subsection{New sensor technology}

Recent advances in sensor technology such as Doppler lidar [7] and other techniques are leading to the development of aircraft-based sensors that will be able to gather large amounts of airflow velocity data in real time. It is likely that aircraft-mounted hardware will soon be available that can accurately scan the area a few hundred feet ahead of the aircraft and sample air particle vector velocities at one-foot intervals or less [8]. With the development of such devices, onboard detection systems that can convey detailed information about airflow hazards to pilots in real time become a possibility.

Consequently, such systems will require an interface that can concisely present large amounts of data to the pilot in real time, yet not distract from the pilot's primary task of flying the aircraft. This is the decision support task we attempt to address in this paper: how does one best present safety-critical information to a cognitively overloaded user in real time?

\section{Initial usability study}

In a preliminary usability study [9], we presented numerous visual representations (using a 3D CAD modeling system and animated video clips) of regions of hazardous airflow to experienced helicopter pilots, while simulating the cockpit view of a helicopter's final approach to shipboard landing on a projection screen. The variables studied included shape, color, and animation of the hazard indicators. A set of images was presented for each of the variables and pilots' verbal commentary was noted.

Common techniques used by flight test engineers in understanding ship airwake usually include 3D motion, such as smoke trails injected into wind tunnels (Figure 5). Viewers of the video sequences often find the visualization of the air particles more instructive than static presentations [10]. However, upon being shown animated imagery over shipboard landing sites, the pilots strongly rejected the use of dynamic indicators.

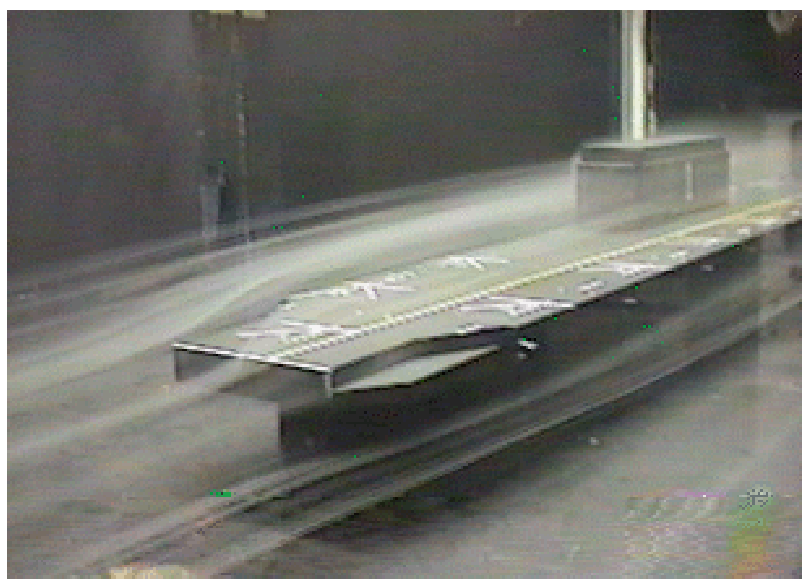

Figure 5. Smoke flow over LHA model in wind tunnel (image courtesy of K. Long)

Pilots favored much simpler imagery than we had initially anticipated. Helicopter pilots landing on shipboard must focus all their attention to complete the landing safely, and have little spare cognition to analyze detailed quantitative information about hazards. An abundance of detail, motion (animation), complex shapes, and too many colors were all ruled out as distracting and possibly dangerous in the high-demand environment. The visual indicators had to be sufficiently translucent so as not to obscure any critical shipboard visual cues that the pilots needed as landing aids. The pilots desired to be informed only of the location of the hazard and its severity-a warning (yellow) or danger (red). In other words, our domain experts had informed us of the need for a decision support system with minimum critical information, not a scientific visualization system, and their reasons had to do with the division of attention in the high-demand environment. 
This first phase of the study also revealed a strong preference by the pilots for a display in which the hazard indicator appears to be spatially conformal with the actual hazard in the physical scene. During potentially dangerous conditions, the pilot's attention will inevitably be focused outside the cockpit during the critical landing moments; he or she will not want to glance away and down at a cockpit instrument display. The pilots strongly favored an augmented-reality hazard visualization display on a headup display (HUD). However, the display must be thoughtfully designed so as not to distract from the key shipboard visual cues, especially when these cues are degraded during a nighttime or poor-weather landing.

Earlier studies have demonstrated that head-up displays with superimposed symbology may occasionally cause performance problems due to attentional capture by the perceptual grouping of the superimposed symbols $[11,12]$. "Scene-linked" head-up displays, or displays where the superimposed symbology appears to lie in the outside scene, can solve this problem. Our study also confirmed the requirement for a head-up display where the hazard indicator is three-dimensional and appears to be physically part of the world.

The pilots stressed the importance of utilizing conventional symbology at all times. They emphasized the danger even a moment of confusion could cause, and strongly recommended that the symbology used in our head-up display conform to current aviation standards. It was particularly important that our symbols not have any chance of being confounded with other types of HUD symbology already in use. The results from this prototype study enabled us to select a design that was substantially different from any existing type of HUD symbology.

\subsection{Visual design of hazard indicators}

For the final version of the system, we designed simple, static, translucent red and yellow surfaces that delineated the outlines of the hazard volumes on shipboard (Figure 6). Yellow indicated caution and red indicated danger. The shape and appearance of the indicators were selected to mark the physical location of the hazard without undue distraction and without duplicating any symbology used for other purposes, while the color meanings are conventional and widely accepted in the aviation world. The boundaries of the hazard regions were determined upon extensive study of the archived airflow data from flight tests and consultation with a Navy flight test engineer. The hazard surfaces were then imported into the visual subsystem of the simulator and linked to the ship so that they appeared to be part of the simulated outside world. The hazard indicators looked like clouds or curtains hovering over the ship (Figure 7). This is an accurate model of shipboard airwake, because any hazardous regions created by wind blowing over ship structures will move along with the ship.

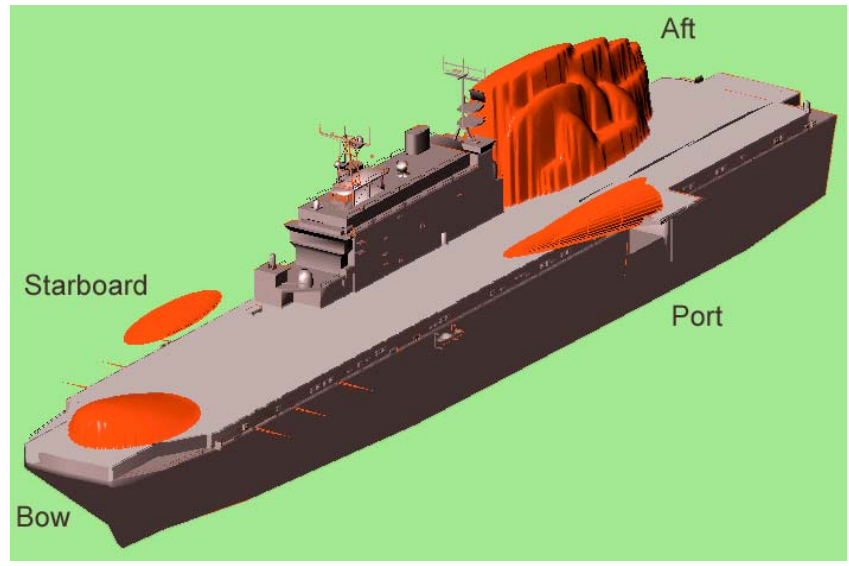

Figure 6. Visual hazard indicators used in the study for the four scenarios, Aft, Bow, Port, and Starboard (actual indicators were more translucent than depicted; see figures 7 and 8).

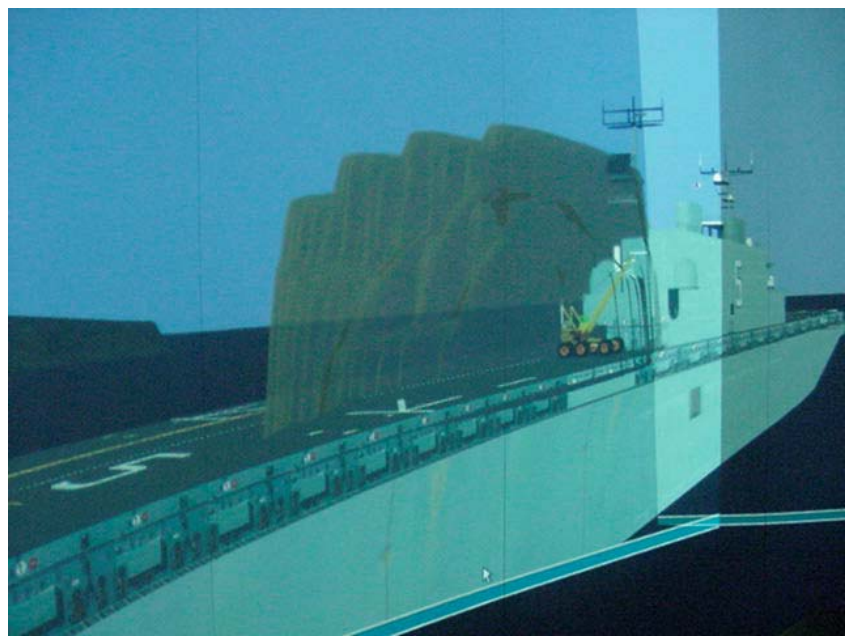

Figure 7. Hazard indicator (yellow, caution) - Image photographed directly from flight simulator screen (lighter vertical area is artifact of photo and does not represent an object in the scene).

\section{Flight simulation study}

To conduct a high-quality usability study of a specialized interface, it is critical that the participants be domain experts. The quality and relevance of the results depend on working with people who actually perform under the demanding conditions that we hoped to duplicate in the study. To test our hypothesis that the presence of a visual hazard indicator could improve helicopter flight safety, we recruited 16 experienced helicopter pilots to participate in the flight simulation study. Each of the 16 pilots flew the same 28 approaches, in varying order, for the within-subjects study. 
The study was performed at Advanced Rotorcraft Technology, Inc. in Mountain View, California, a small flight simulation company specializing in rotorcraft nonlinear dynamics modeling. ART's aerodynamic models have been verified by the US Navy via stability and control techniques and frequency domain validation [13, 14], and Navy flight test engineers and pilots have stated that they are more aerodynamically accurate than other rotorcraft flight simulators currently available [14].

Airflow data from computational fluid dynamics [15] calculations and shipboard flight tests were input into the simulator. The pilots sat in an aircraft seat with full helicopter controls (cyclic, collective, and tail rotor pedals) with force feedback, in front of a cockpit instrument panel. They viewed a three-channel projection outside world visual system with a 188 " horizontal by 54 " vertical field of view on a 6.5 -ft radius cylindrical screen (Figure 8 ).

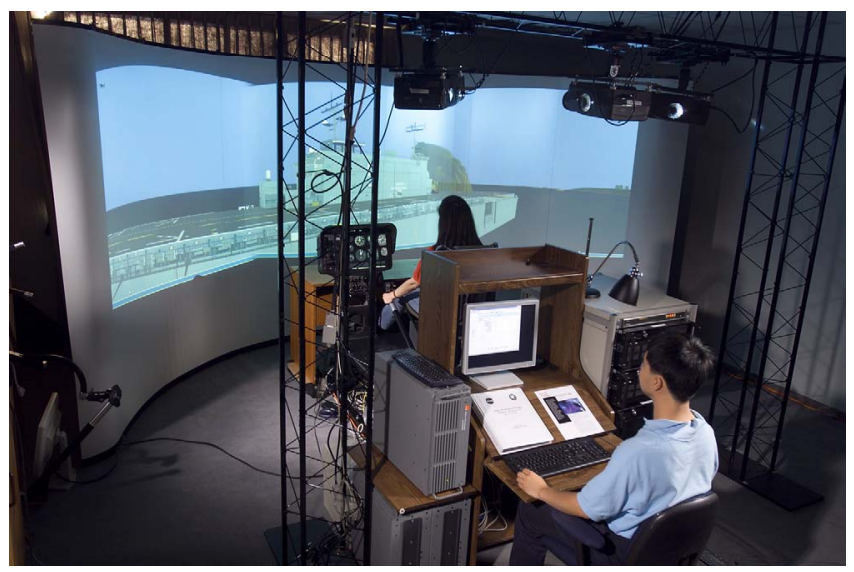

Figure 8. ART flight simulator with pilot in front of projection screen and operator at rear console

The pilots flew simulated final approaches to land a Sikorsky H-60 helicopter on a moving ship (an LHA or "Tarawa-class" Navy amphibious assault ship) under different wind conditions, some of which included airflow hazards such as vortices, downdrafts, or turbulence on or near the landing site. Four different landing difficulty levels were used, based on the US Navy's Pilot Rating Scale of landing difficulty [6]. Other than the control approaches (where there was no hazardous airflow), each approach was flown twice by each pilot, once with a hazard indicator present and once without. Data was collected both objectively from the flight simulator's recording capability and subjectively from a Likert-scale questionnaire administered to the pilots after the flight.

\subsection{Dependent variables}

During the simulation, variables such as velocity and position of aircraft in $\mathrm{x}, \mathrm{y}, \mathrm{z}$, control stick position both lateral and longitudinal, collective and pedal positions, landing gear forces, etc., were collected by the flight simulator at $10 \mathrm{~Hz}$ and stored in data files labeled for each run and pilot. However, our primary dependent measure was the crash rate. A "crash" was defined as an impact with the ship's deck with a vertical velocity of 12 feet per second or greater as measured by the simulator. In order to be certified for shipboard use in the US Navy, rotorcraft must be able to withstand an impact of $12 \mathrm{fps}$ at touchdown [16].

We also gathered subjective pilot opinions from a 21probe Likert-scale (1-5) questionnaire administered to the pilots at the end of the simulation. For each probe, the pilots had to circle one of "Strongly Disagree" (1), "Disagree" (2), "Neither Agree Nor Disagree" (3), "Agree" (4), and "Strongly Agree" (5). This questionnaire included probes concerning the distractibility of the indicators and pilot acceptance and probable use of the system in flight.

\section{Results}

The study results [17] showed that use of the hazard visualization system leads to a statistically significant decrease in crash rate for a critical class of landings - those where landing is permitted, but hazardous.

The mean crash rate for this class of landings when no hazard indicator was displayed was 19\% (with a standard error of .049). When a hazard indicator was displayed, the mean crash rate dropped to $6.3 \%$ (with a standard error of .031). A t-test for paired samples showed that the hypothesis that the presence of the hazard indicator reduces the frequency of crashes during simulated shipboard helicopter landings was confirmed $(\mathrm{t}=2.39, \mathrm{df}=63$, $\mathrm{p}=0.00985$ ).

A more detailed description of the study, its results and conclusions can be found in [17]. The study results were sufficiently strong to indicate that the system should improve helicopter flight safety under hazardous conditions. Further indications were that this type of hazard indicator did not unduly distract the pilots from their primary task (pilot performance was not degraded by the presence of the indicator), and that the presence of the visual system made landing the helicopter under hazardous conditions equivalent to landing under calm conditions (as demonstrated by pilot performance on the control approaches in the study). Finally, $81 \%$ of the pilots in the study said they would use this display system if it were available on their aircraft, thus confirming the probable efficacy of a cockpit decision support system such as described in this paper.

\section{Conclusions and future work}

For a real time cockpit decision support system where pilots must make time-critical safety decisions under stress 
and where the display is peripheral to the primary task, our studies have demonstrated that simple and static visualizations yield improved performance on safetycritical tasks. In this experiment, we discovered that the type of visualization needed to improve operational safety was much simpler than that needed for analysis of airflow hazards, providing an example in which the appropriate visualization differs for analysis vs. presentation.

Due to human limitations on attentional bandwidth and processing capacity, decision support systems for pilots must provide a terse summary of critical information that can be evaluated rapidly. Static visual displays are wellsuited for this function, but care must be taken to use appropriate symbology, to avoid attentional capture or distraction, and to conduct usability studies with domain experts to ensure that the final interface design satisfies all these constraints. Additional challenges exist in the implementation of a visual display system utilizing scenelinked imagery, such as synchronizing the static hazard indicator with the moving scene outside.

It is also important to develop objective metrics to evaluate such visual decision support systems. Pilots may prefer a display that does not produce improved performance. Due to the compelling nature of 3D imagery in visual displays, subjective preference does not always lead to superior performance [18]. It was for this reason that we conducted the usability study in a highly realistic flight simulator that had the capability of objectively measuring pilot performance, including aircraft position, velocity, and landing gear forces at touchdown. Further studies of visualization systems should be conducted to confirm these principles.

\section{Acknowledgments}

We gratefully acknowledge the support of Kurtis R. Long of the Navy Dynamic Interface Flight Test Group, and the staff of Advanced Rotorcraft Technology, Inc. This work was funded by NASA Ames Research Center.

\section{References}

[1] G. F. Switzer and C. L. Britt, "Performance of the NASA Airborne Radar with the Windshear Database for Forward-Looking Systems," NASA CR 201607, 1996.

[2] L. Wallace, "Airborne Trailblazer," NASA Langley Wind Shear Program, NASA Langley, http://oea.larc.nasa.gov/trailblazer/SP-4216/, 1993, accessed 2004.

[3] FAA National Aviation Safety Analysis Center, https://www.nasdac.faa.gov/, accessed 2004.

[4] C. H. Wilkinson, S. J. Zan, N. E. Gilbert, and J. D. Funk, "Modelling and Simulation of Ship Air Wakes for Helicopter Operations," RTO-MP-15, 1999.
[5] M. Mewhinney, "Flow over helicopter carriers," NASA Ames Astrogram, October 1998.

[6] S. L. Williams and K. R. Long, "Dynamic Interface Flight Tests and the Pilot Rating Scale," Proc. American Helicopter Soc. 53rd Annual Forum, 1997.

[7] NASA Marshall Space Flight Center Lidar Tutorial, http://www.ghcc.msfc.nasa.gov/sparcle/sparcle tutorial.ht $\underline{\mathrm{ml}}$, accessed 2004.

[8] K. Asaka, S. Kameyama, T. Ando, Y. Hirano, H. Inokuchi, and T. Inagaki, "A 1.5 um all-fiber pulsed airborne Doppler lidar system," JAXA IS17-06, 2003.

[9] C. Aragon, "Usability Evaluation of a Flight-Deck Airflow Hazard Visualization System," Proc. 23rd Digital Avionics Systems Conference, Salt Lake City, UT, USA, 2004.

[10] S. L. Williams and K. R. Long, "ADS-33 and Shipboard Rotorcraft Operations: A USN Flight Test and Simulation Perspective," Proc. American Helicopter Society 53rd Annual Forum, Virginia Beach, VA, 1997.

[11] R. S. McCann and D. C. Foyle, "Scene-linked symbology to improve situation awareness," Proc. Aerospace Medical Panel Conference on Situation Awareness, 1995.

[12] L. Prinzel, "Head-Up Displays and Attention Capture," NASA Technical Memorandum 2004-213000, 2004.

[13] C. He and J. Goericke, "FLIGHTLAB UH-60L Simulation Model Validation," Army ART TR 2011 DAAH10-03-C-0001, Nov. 2004.

[14] K. Strope, C. Borden, and J. Harding, "Verification and Validation of a UH-60 FLIGHTLAB model in support of the UH-60M Limited User Test," Proc. American Helicopter Society 60th Annual Forum, Baltimore, MD, 2004.

[15] Polsky, S., 2003, "CFD Prediction of Airwake Flowfields for Ships Experiencing Beam Winds", AIAA Paper 2003-3657, 21st AIAA Applied Aerodynamics Conference, Orlando, FL.

[16] US Navy, "NATOPS Flight Manual - Navy Model SH-60B Aircraft," US Navy A1-H60BB-NFM-000, 1 March 2004.

[17] C. Aragon and M. Hearst, "Improving Aviation Safety with Information Visualization: A Flight Simulation Study," CHI 2005: ACM Conference on Human Factors in Computing Systems, Portland, OR, 2005.

[18] H. S. Smallman, M. St. John, H. M. Oonk, and M. B. Cowen, "Information availability in 2D and 3D displays," IEEE Computer Graphics and Applications, vol. 21, pp. $51-57,2001$. 